\title{
Effects of JPEG and JPEG2000 Lossy Compression on Remote Sensing Image Classification for Mapping Crops and Forest areas
}

\author{
Alaitz Zabala, Xavier Pons \\ Department of Geography \\ Autonomous University of Barcelona, UAB \\ Cerdanyola del Vallès, Spain \\ Alaitz.Zabala@uab.es, Xavier.Pons@uab.es
}

\author{
Ricardo Díaz-Delgado \\ Remote Sensing \& GIS Laboratory \\ Estación Biológica de Doñana-CSIC \\ Sevilla, Spain \\ rdiaz@ebd.csic.es
}

\author{
Fernando García, Franscesc Aulí-Llinàs, Joan Serra-Sagristà \\ Dept. of Information and Communications Engineering, DICE \\ Autonomous University of Barcelona, UAB \\ Cerdanyola del Vallès, Spain \\ fgarcia@abra.uab.es, Francesc.Auli@uab.es, Joan.Serra@uab.es
}

\begin{abstract}
This study measures the effect of lossy image compression on the digital classification of crops and forest areas. A hybrid classification method using satellite images and other variables has been used. The results contribute interesting new data on the influence of compression on the quality of the produced cartography, both from a "by pixel" perspective and regarding the homogeneity of the obtained polygons. The classified area in classifications only carried out with radiometric variables or with NDVI and humidity (for crops) increases as image compression increases, although the increase is smaller for JPEG2000 formats and for crops. On the other hand, the classified area decreases in classifications which also take into account topoclimatic variables (for forests). Overall image accuracy diminishes at high compression ratios (CR), although the point of inflection occurs at different $C R$ depending on the compression format. As a rule, the JPEG2000 format gives better results quantitatively for forests (accuracy and classified area) and visually (images with less "salt and pepper" effect) for both land covers.
\end{abstract}

Keywords: Remote sensing; image classification; lossy compression; natural areas; crop areas

\section{INTRODUCTION AND AIMS}

In 1991 the JPEG format [1], developed by the Joint Photographic Experts Group, first appeared and revolutionized image compression due to the fact that it achieves very high compression ratios with no appreciable loss of image quality, at least for file sizes up to approximately $20 \%$ of the original. Later there appeared compression techniques based on wavelets transformations which permit even higher compression ratios with similar image quality. In recent years, SID, ECW and JPEG2000 formats [2] have been particularly popular with the TD and SIG community. It is important to bear in mind that in every case we are dealing with lossy compression algorithms, which sacrifice part of the data in order to achieve a higher compression ratio.

In spite of the spectacular nature of the compression ratios achieved, there has been little quantitative analysis of the implications of these compressions. This study aims to assess the influence of image compression on digital classification applied to areas of natural vegetation based on the authors' own experience and on that of studies [3], [4] and [5].

Moreover, our study covers a wider area, which should provide more representative results. In line with current practice, it also aims to evaluate multitemporality and the use of topoclimatic or humidity and NDVI variables to improve the classification.

\section{MATERIAL AND METHODS}

The classification method used is a combination of satellite images and other variables over vegetation land covers, which is designed to improve the accuracy of the classifications [6].

The training areas were obtained from the Mapa d'Hàbitats de Catalunya (Habitat Map of Catalonia) and underwent statistical treatment to guarantee their quality.

For forest areas, in order to measure the effect of topoclimatic variables and compression on the results of the classification, various scenarios were considered: scenario $\mathrm{R}$ had only images (radiometric variables) whereas scenario RTC also had topoclimatic variables and NDVI. For crop areas, humidity and NDVI variables were used to improve classification; therefore two scenarios were also considered: scenario $\mathrm{R}$ had only images (radiometric variables) whereas scenario RHN also had humidity and NDVI variables. On both type of land cover and for each scenario JPG and JPEG2000 (referred as $\mathbf{J} \mathbf{2 K}$ ) compression techniques were analyzed.

It would not have been possible to carry out this study without the financial assistance of the Ministry of Science and Technology and the FEDER funds through the research project: "Wavelet image compression for Remote Sensing and GIS applications" (TIC2003-08604-C04). We would like to express our gratitude to the Catalan Water Agency and to the Department of the Environment and Housing of the Generalitat (Autonomous Government) of Catalonia for their investment policy and the availability of Remote Sensing data, which has made it possible to conduct this study under optimal conditions. We would also like to thank our colleagues of the Department of Geography, CREAF and the CCD of the UAB who have collaborated in any way in the treatment of the images, and INTA for its efficient image subscription service. 
Compression size was based on compression ratios (CR) and not on compression quality (standard for JPG) since we considered it more relevant given the clearly practical applications of our research. It should be borne in mind that the same compression ratio may produce different degrees of quality depending on the type of image. CR is computed as follows:

$$
C R=\frac{\text { Size of Compressed File }}{\text { Size of Original File }}
$$

Analysis of classification accuracy (with or without compression) is based on test areas (ground-truth layer) which are different from the training areas.

\section{A. Areas and scenes used}

Two medium-sized areas with different levels of spatial fragmentation were chosen for each land cover and were analyzed using Landsat images.

The first forest area is the Garrotxa county $(1264 \times 1264$ pixels of 20x20m, 50176 ha of forests) and was analyzed using 12-03-03, 26-04-02, 13-06-02 and 16-08-02 images. The second forest area, which is more fragmented, is the MaresmeVallès county (3474x2323 pixels of 20x20m, 146687 ha of forests) and was analyzed using 12-03-03, 13-06-02, 26-07-03 and 11-08-03 images.

Regarding crops, two areas were selected from the Lleida region. Dimensions of the first area are $1517 \times 1311$ pixels of 20x20m (53585 ha of crops) and dimensions of the second one, more fragmented, are 1307x1059 pixels of 20x20m (50099 ha of crops). Both were analyzed using 16-05-04, 17-06-04, 1907-04, 23-10-04 and 08-11-04 images.

A mask obtained from the Mapa del Cobertes del Sòl de Catalunya (Land Cover Map of Catalonia) was applied over the original images (after they had been geometrically and radiometrically orthocorrected) in order to classify only the areas of forest or crops, respectively.

\section{B. Image compression/decompression}

The compression/decompression algorithms used were the implementation of the MiraMon v.5.2 classic JPEG based on the JPEG public libraries (JPEGIMG module) and the JPEG2000 implementation of the DICE (BOI software).

JPEG compression format uses byte type images, for this reason it was considered necessary to carry out the whole battery of tests in byte format, and the original images were converted into this format before compression by means of linear stretch.

\section{a) NODATA value}

The original images display areas without data (NODATA) due to the radiometric corrections to which they were subjected and the presence of a small number of clouds. Not all the compression/decompression programs used are currently able to recognize these NODATA values. Using them as actual values when compressing will generate important errors in the images generated. It is therefore necessary to eliminate these values from the images before compression. Elimination is carried out using the MiraMon FagoVal module, which selectively eliminates (phagocytes) a given value in raster files, replacing it (in this case) by the arithmetical mean of the adjacent values. Finally, it is necessary to create a mask with the NODATA areas in the original images in order to reapply it over the image after compression.

\section{b) JPEG and JPEG2000 compression}

In the classic JPEG format, the quality of the resulting JPEG file is usually set. In general, and even after modifying the quality, it is not possible to generate a JPEG file of a given size (in other words, one whose compression ratio with respect to the original file is a concrete value). Therefore, for each compression scenario the JPEG file whose size is nearest to the one that is necessary to obtain this compression ratio was chosen.

The standard JPEG2000 includes the possibility of generating a resulting image of a specific size. It is therefore a simple matter to generate compressed images with a given compression ratio.

\section{c) Decompression and subsequent treatment}

After decompressing the images, it is necessary to eliminate the 255 value (future NODATA value) assigning it to the nearest value immediately below. Then, the mask corresponding to the original NODATA areas must be applied to each image.

\section{Classification}

The classification methodology employed allows the variables used in the classification to be standardized; this process was applied to forest areas. The hybrid classification is subsequently carried out using the MiraMon IsoMM and ClsMix modules (Fig. 1). The classification parameters which maximize the accuracy in the forest or crops uncompressed images classification are used for all scenarios.

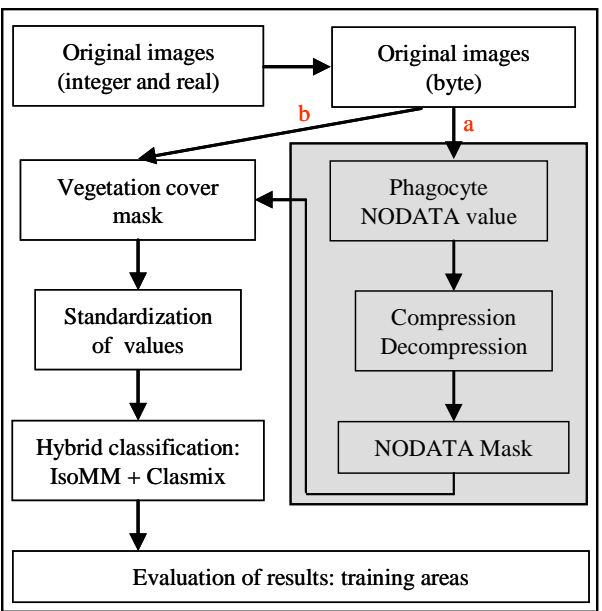

Figure 1. Diagram of methodology used. a) workflow for non-compressed images; b) workflow for compressed images

\section{RESULTS}

Figures 2 and 3 show the results for forests and crops areas respectively. For each land cover two sub-areas were analyzed. 

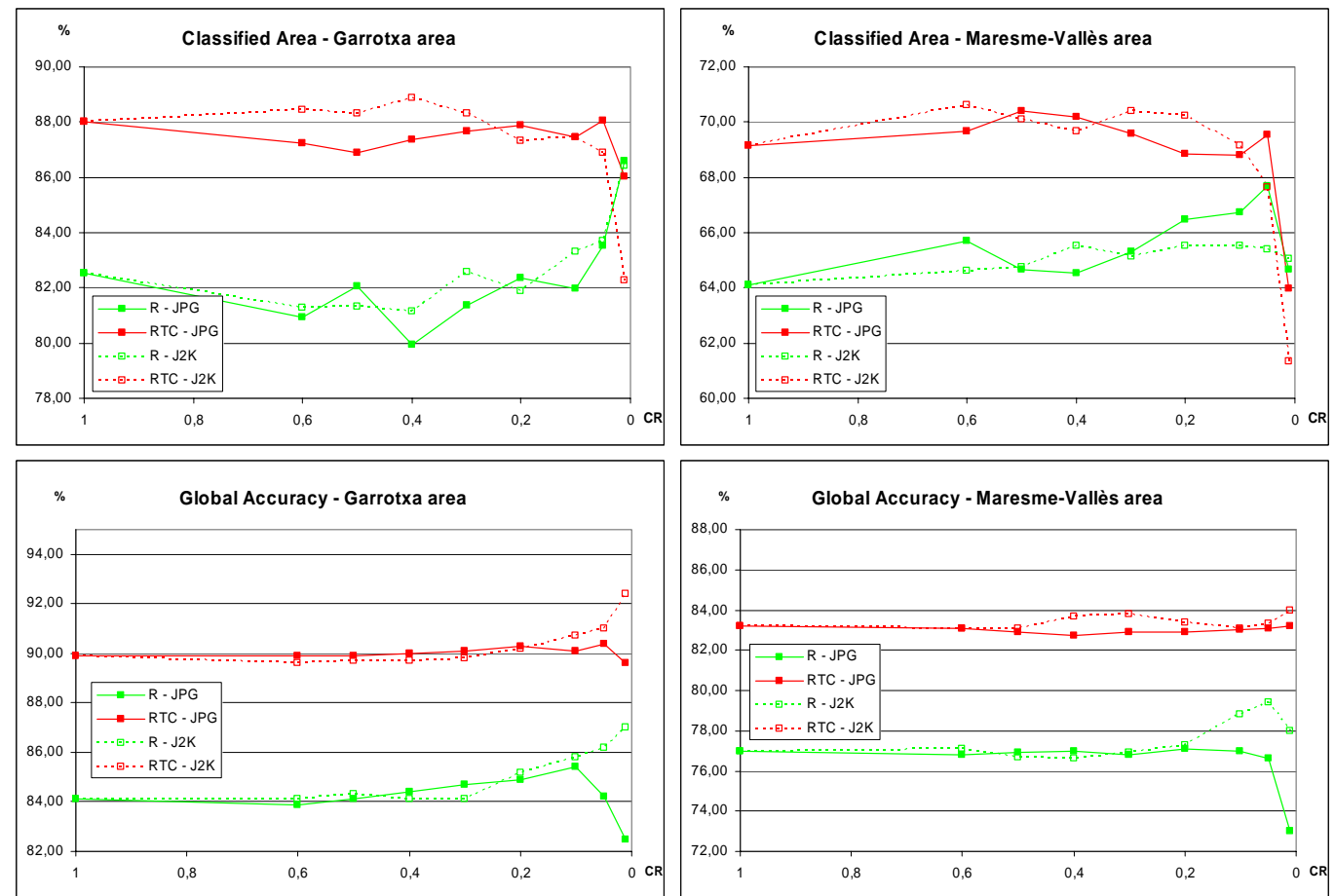

Figure 2. Forest areas results: Classified area (top graphs) and Global accuracy (bottom) for Garrotxa (left graphs) and Maresme-Vallès (right) areas
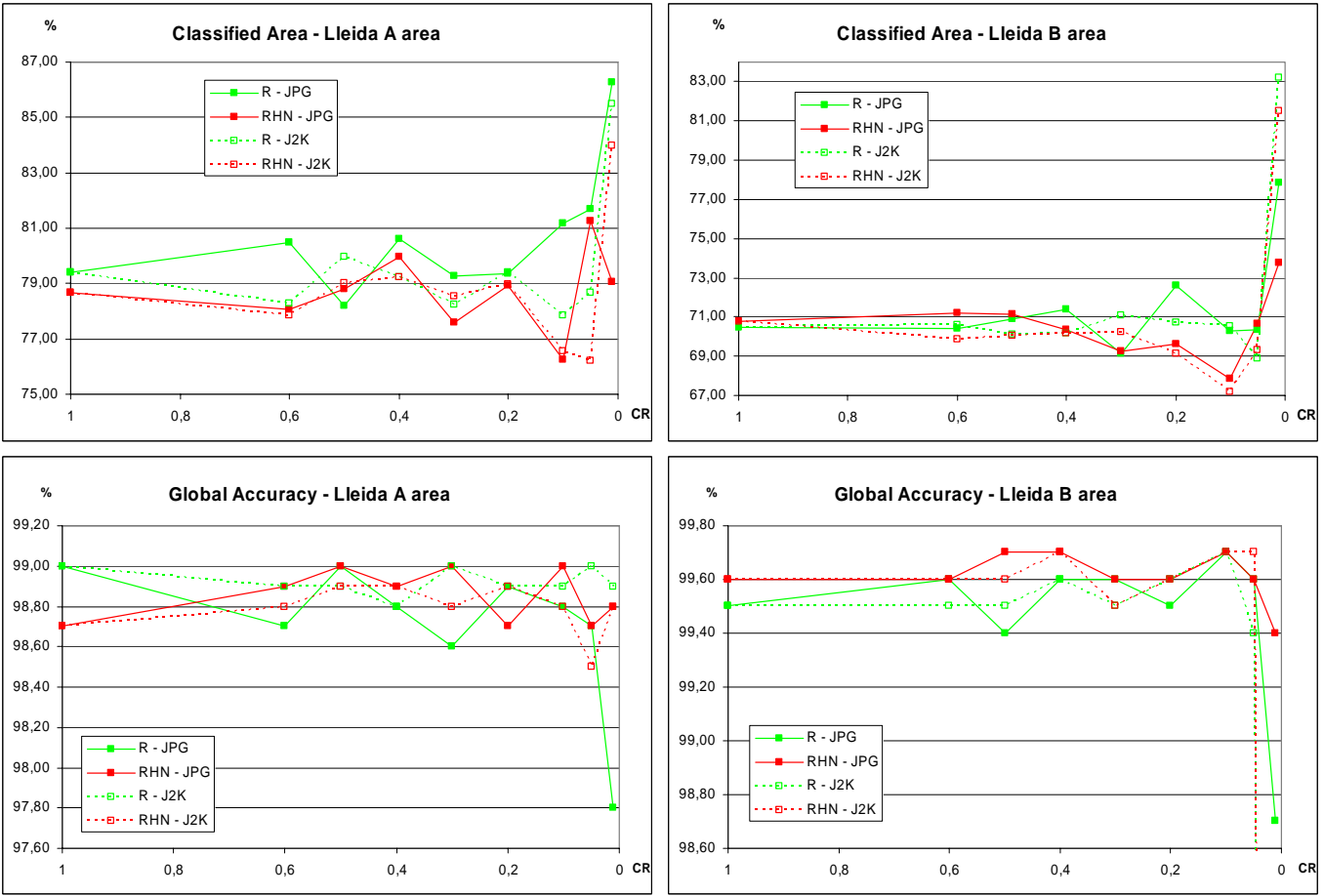

Figure 3. Crop areas results: Classified area (top graphs) and Global accuracy (bottom) for Lleida A (left graps) and Lleida B (right) areas

For each sub-area the top graph shows the percentage of classified area obtained by the classifications while the bottom graph indicates the global accuracy, both according to the compression ratio (CR). Graphs of less fragmented areas (Garrotxa and Lleida A) are on the left.

In all graphs, green color indicates classifications that only include radiometry (scenario $\mathrm{R}$ ) while red color those that also include other variables (scenarios RTC and RHN). Continuous line: JPG compression; dotted line: J2K compression.

As the CR decreases, in scenario R the tendency is for the classified area to increase. This is probably due to a beneficial homogenization of the images when compressed. The increase in area is smaller for the $\mathrm{J} 2 \mathrm{~K}$ (forests and crops) and for crop areas (JPG and J2K). In scenario RHN (crops) classified area 
also increases with CR. Only in scenario RTC (forest areas), classified area decreases. This would seem to indicate that compression affects the topoclimatic variables more heavily, perhaps because they are more continuous.

As CR decreases, the global accuracy increases at first, but decreases for JPG at low CR, specially in scenario R - JPG. In scenario RTC - JPG or RHV - JPG, accuracy also decreases but in a lesser degree. This tendency is observed for both forests and crops but is clearer in forest areas because of the higher variability results of crop areas. On the other hand, for the J2K format, global accuracy appears to increase indefinitely for the Garrotxa area, but nor for the Maresme-Vallès area, neither for both crop areas.

\section{CONCLUSIONS}

Conclusions suggest the following optimal work scenarios:

- Scenario R - JPG: CR 0.2-0.1 is optimal for less fragmented images (Garrotxa and Lleida A), as accuracy is greater (higher than the original image) and the percentage of classified area is similar or only slightly smaller for forests, while accuracy is slightly smaller but classified area is larger for crops. For more fragmented images, optimal CR is between 0.1-0.05 for forests and 0.1 for crops.

- $\quad$ Scenario R - J2K: for less fragmented images we have not reached the optimal CR limit in forests (in any CR, accuracy and classified area increase). Regarding crops less fragmented areas, CR is 0.05-0.01 while for more fragmented images CR is 0.05 (forest) and 0.1 (crops).

- Scenario RTC - JPG: optimal CR for slightly fragmented images is 0.2 for forests and 0.05 for crops. For forests the results are similar to those obtained from the original images. For crops the classified area is larger than the obtained with the original images. In more fragmented images, CR 0.5 has the largest classified area and only slightly less accuracy for forests and larger accuracy for crops.

- $\quad$ Scenario RTC- J2K: for less fragmented images CR is 0.3-0.2 (forest) and 0.4 (crops). For more fragmented images, CR is 0.3 for forest and 0.4 for crops.

It is important to point out that according to classification results, J2K format is better than JPG for forests but not for crops. Nevertheless, according to visual effects, classification obtained from J2K compressed images has less "salt and pepper effect” therefore, and from a cartographic perspective, J2K approach is much more effective for both (Fig. 4).

In accordance with the presented results, a general trend may be set about greater sensibility of crops to compression, thus they have to be preserved from heavy compression.

It is also important to point out that in borders between highly differentiated spectral classes compression produces mixing effects that lead to errors in these areas. Fig. 5 shows that in the J2K classification limits among alfalfa (yellow) and winter cereals (dark blue) and maize (clear blue) turn up fruit trees (red).

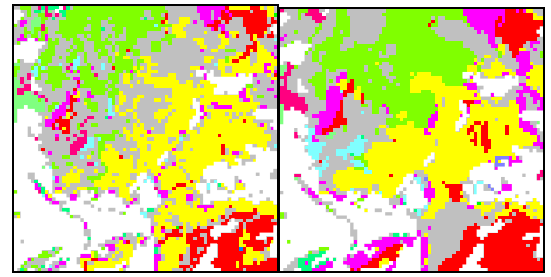

Figure 4. Detail of forest classification: original (left), J2K CR 0.01 (right)

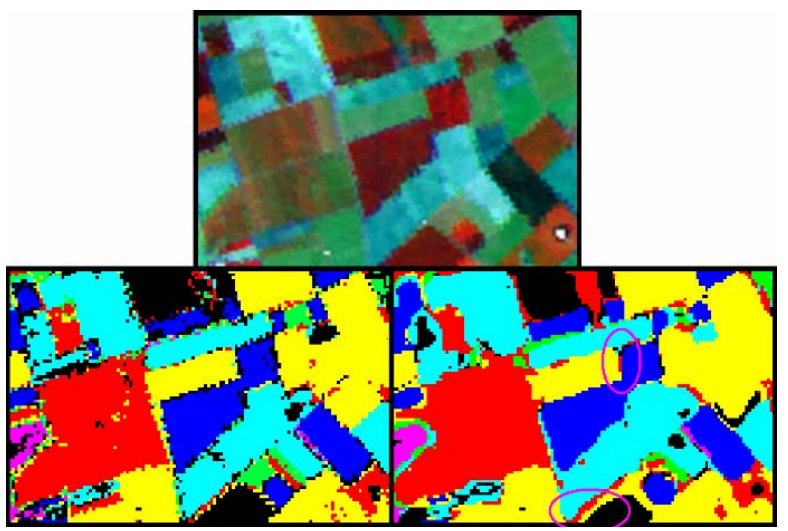

Figure 5. Border effects (on crops): 4, 5, 3 RGB-composition (top), original classification (left), J2K CR 0.01 classification (right).

These border effects will have a marked impact on future studies of changes in land use which may produce masked results due to the erroneous classification of the border areas. This is aggravated by the virtual non-existence of test points in these areas, which will hide the decrease in global accuracy.

Future work will be aimed to obtain more results to better define the relationship between CR and accuracy or classified area, specially for crop areas. The same aim may be achieved studying accuracy and classified area results for different sets of classifications using other parameters. Finally another accuracy estimation method may be also tested.

\section{REFERENCES}

[1] ISO/IEC 10918-12, “Digital compressing and coding for continuoustone still images”.1994.

[2] ISO/IEC 15444-1, JPEG 2000 image coding system - Part 1: Core coding system. 2000.

[3] C. Pérez, D. Aguilera, A. Muñoz, "Estudio de viabilidad del uso de imágenes comprimidas en procesos de clasificación”. In R. Pérez Utrero, P. Martínez Cobo. Teledetección y desarrollo regional. X Congreso Nacional de Teledetección, 2003, pp. 309-312.

[4] F. Tintrup, F. De Natale, D. Giusto, "Compression algorithms for classification of remotely sensed images”. Acoustics, Speech and Signal Processing. ICASSP '98. Proceedings of the 1998 IEEE International Conference, Vol. 5, 1998, pp. 2565-2568.

[5] J.D. Paola, R.A. Schowengerdt, "The effect of lossy image compression on image classification”. Geoscience and Remote Sensing Symposium, 1995. IGARSS '95. 'Quantitative Remote Sensing for Science and Applications', International 1: 118-120.

[6] P. Serra, X.Pons, D. Saurí, "Post-classification change detection with data from different sensors. Some accuracy considerations”. Int. J. Remote Sens., Vol. 24, No. 16, 2003, pp.3311-3340. 